\title{
Fourier Analysis in Several Parameters
}

\section{R. Fefferman}

Dedicated to my dear friend and teacher, Professor Alberto P. Calderón

Clearly, one of the most basic contributions to the fields of real variables, partial differential equations and Fourier analysis in recent times has been the celebrated theorem of Calderón and Zygmund on the boundedness of singular integrals on $R^{n}$ [1]. One way to state this result is as follows:

Theorem. Suppose T is a bounded linear operator on $L^{2}\left(R^{n}\right)$. Suppose further that whenever $a(x)$ is a function supported on a cube $Q$ satisfying $\int_{Q} a(x) d x=0$, then $-T$ satisfies

$$
\int_{c_{\widetilde{Q}}}|T(a)(x)| d x \leqslant C\|a\|_{L^{1}(Q)},
$$

where $\tilde{Q}$ denotes the double of $Q$. Then $T$ is of weak type on $L^{1}\left(R^{n}\right)$, i.e. for each $\alpha>0$ and $f \in L^{1}\left(R^{n}\right)$,

$$
\left|\left\{x \in R^{n}|| T(f)(x) \mid>\alpha\right\}\right| \leqslant \frac{C}{\alpha}\|f\|_{L^{1}\left(R^{n}\right)} .
$$

From here, as everyone knows, by interpolation, we may obtain $L^{p}$ results when $1<p \leqslant 2$. The importance of this result goes beyond what is apparent from the statement of the theorem. Rather, in the proof, we clearly see the role of the Hardy-Littlewood Maximal operator, and how this operator controls classical singular integrals. In a great number of problems in higher dimensions, the Hardy-Littlewood operator no longer suffices to control the operator at hand, and one is forced to deal with more complicated maximal functions. Let us give some basic examples from the theory of Fourier integrals in $R^{n}$. In each case, just as in the classical Calderón-Zygmund case, a particular 
maximal operator will be considered which controls the singular integral in question. Somewhat later, we shall consider the question of how to prove estimates on these maximal operators.

If $f(x)$ is a function on $R^{n}$ with Fourier transform $\hat{f}(\xi), \xi \in R^{n}$, then in some sense the central concern of Fourier analysis should be to determine in which sense we have

$$
f(x)=\int_{R^{n}} \hat{f}(\xi) e^{i \xi \cdot x} d \xi .
$$

There are a number of ways in which we can form the partial sum operators corresponding to this Fourier integral. First we can take «rectangular partial sums» given by

$$
S_{r_{1}, r_{2}, \ldots, r_{n}} f(x)=\int_{\substack{\left|\xi_{i}\right|<r_{i} \\ i=1,2, \ldots, n}} \hat{f}(\xi) e^{i \xi \cdot x} d \xi .
$$

It is easy to see that for many purposes, just as in one dimension the partial sum operators are controlled by the Hilbert transform, the rectangular partial sums are best understood in terms of the multiple Hilbert transform, $H_{1} H_{2} \cdots H_{n}$ given by

$$
H_{1} H_{2} \cdots H_{n} f=f * \frac{1}{x_{1} x_{2} \cdots x_{n}} .
$$

This singular integral, although bounded on all the spaces $L^{p}\left(R^{n}\right)$ for $1<p<\infty$ is very different from the classical Calderón-Zygmund operators on $R^{n}$. The kernel of this multiparameter singular integral has singularities on the coordinate hyperplanes, unlike the Calderón-Zygmund kernels, which are smooth away from the origin. As a result, the singular integrals like the multiple Hilbert transform are not weak type on $L^{1}\left(R^{n}\right)$. The maximal operator which is relevant to the study of operators like $H_{1} H_{2} \cdots H_{n}$ is the JessenMarcinkiewicz-Zygmund strong maximal function, $M_{S} f$, defined at a point $x \in R^{n}$ by

$$
M_{S} f(x)=\sup _{x \in R} \frac{1}{|R|} \int_{R}|f(y)| d y,
$$

where the sup is taken over all rectangles $R \subseteq R^{n}$ whose sides are parallel to the coordinate axes and contain $x$. As we would expect, $M_{S}$ is not weak type on $L^{1}$, but rather satisfies

$$
\left|\left\{x \in R^{n}|| x \mid<1, M_{S} f(x)>\alpha\right\}\right| \leqslant \frac{C}{\alpha}\|f\|_{L(\log L)^{n-1}(|x|<1)} .
$$


We shall consider singular integrals and maximal functions like $H_{1} H_{2} \cdots H_{n}$ and $M_{S}$ later in this article. They are important because in some sense they are the simplest examples which commute with a multiple parameter family of dilations, and hence are the best understood. Next, suppose we consider the spherical partial sum operators:

$$
S_{r}(f)(x)=\int_{|\xi|<r} \hat{f}(\xi) e^{i \xi \cdot x} d \xi, \text { where }|\xi|=\left(\sum_{i=1}^{n} \xi_{i}^{2}\right)^{1 / 2} .
$$

Although one might be tempted to guess, from the presence of the single parameter $r$, that the convergence of the $S_{r} f$ in $L^{p}$ should be a consequence of the theory of the classical Calderón-Zygmund singular integrals and maximal functions, this is not at all the case. Rather the operator $S_{r}$ is much more complicated. As Charles Fefferman [2] has shown, the operator $S_{r}$, which in one dimension is bounded on all $L^{p}$ for $1<p<\infty$, is unbounded on all $L^{p}\left(R^{n}\right)$ whenever $n>1$ and $p \neq 2$. The reason for the bad behavior of $S_{r}$ is that the controlling maximal operator is the multiparameter «Kakeya maximal operator», $M_{K}$ defined by

$$
M_{K} f(x)=\sup _{x \in R} \frac{1}{|R|} \int_{R}|f(y)| d y,
$$

where the supremum is taken over all rectangles (of arbitrary orientation) containing $x$. This operator is unbounded on all $L^{p}$ spaces whenever $p<\infty$. In order to have a theory of operators which resemble the multiplier $T$ given by $T \hat{f}(\xi)=\chi_{|\xi|<1} \cdot \hat{f}(\xi)$, but which has interesting positive results about $L^{p}$ boundedness, we may look in two directions.

First, we may view the negative results about $T$ to be a consequence of the lack of smoothness of the multiplier $\chi_{|\xi|<1}$. We may ask what happens when we replace $\chi_{|\xi|<1}$ by its smooth analogue $\chi_{|\xi|<1} \cdot\left(1-|\xi|^{2}\right)^{\alpha}=m_{\alpha}(\xi)$, to get the Bochner-Riesz operator of order $\alpha, T_{\alpha}$ given by

$$
T_{\alpha} \hat{f}(\xi)=m_{\alpha}(\xi) \cdot \hat{f}(\xi),
$$

when $\alpha>0$.

Now, these operators, at least in $R^{2}$, are controlled by a maximal function of the Kakeya type, but somewhat more tame. According to the work of Carleson-Sjölin, [3] C. Fefferman [4] and Córdoba [5], $T_{\alpha}$ is a bounded operator on $L^{p}\left(R^{2}\right)$ when $4 / 3 \leqslant p \leqslant 4$ and $\alpha>0$. Fefferman and Córdoba explain this by introducing real variable machinery centered around the maximal operators

$$
\mathfrak{M}_{N} f(x)=\sup _{x \in R \in R_{N}} \frac{1}{|R|} \int_{R}|f(y)| d y
$$


where $R_{N}$ denotes the set of all rectangles whose eccentricity does not exceed $N$. Thus, as $N \rightarrow \infty$, in some sense $\mathfrak{T}_{N} \rightarrow M_{K}$ and so of course the $\mathfrak{T}_{N}$ could not be uniformly bounded. However, for $p \geqslant 2$, in $R^{2}$ the operator norm of $\Re_{N}$ grows only like a power of $\log N$ as $N$ becomes large, while if $p<2$ its norm grows like a power of $N$. It turns out that the behavior of $\mathfrak{T}_{N}$ on $L^{2}\left(R^{2}\right)$ controls that of $T_{\alpha}$ on $L^{4}\left(R^{2}\right)$. Below, we shall see that the properties of $\mathfrak{T I}_{N}$ on the critical space $L^{n}\left(R^{n}\right)$ for $n \geqslant 3$ are not at this time understood, and so we also do not know the $L^{p}$ boundedness of certain of the $T_{\alpha}$ in higher dimensions. This is one of the important open questions of the multiple parameter theory.

There is also another way of recapturing a set of positive results analogous to the negative ones for the disk multiplier. Since $\chi_{|\xi|<1}$ is not a multiplier on $L^{p}\left(R^{2}\right)$ except when $p=2$, it is natural to ask for a characterization of those sets $S \subseteq R^{2}$ for which $\chi_{S}(\xi)$ is a multiplier. Charles Fefferman's counterexample to the disk conjecture shows that for a «nice» open set $S$ whose boundary is curved, there are no non-trivial multiplier results on $L^{p}$ for $\chi_{S}$. This leads to the remaining case in which the boundary of $S$ consists of line segments oriented in certain directions $\theta_{k}, k=1,2, \ldots$. Córdoba and the author [6] have proven roughly that if one considers the maximal operator $\mathfrak{N}_{\theta}$ corresponding to the class of rectangles oriented in one of the directions $\theta_{k}$, then $\chi_{S}$ is bounded on $L^{p}\left(R^{2}\right)$ if and only if $\Re_{\theta}$ is bounded on $L^{(p / 2)^{\prime}}\left(R^{2}\right)$ (here $p \geqslant 2$ ). Notice that under the correspondence $p \rightarrow(p / 2)^{\prime}$ the $L^{4}$ result for multipliers corresponds to an $L^{2}$ result for maximal functions, which suggests that the pattern of dependence discussed above of $T_{\alpha}$ on $\mathfrak{T}_{N}$ is valid in more general contexts. The theme of Calderón and Zygmund that the boundedness properties of multipliers and singular integrals are governed by those of maximal operators hast thus been extended in may cases to the multi-parameter setting. Naturally, it becomes very important, in light of this, to prove maximal theorems. Although as recently as about ten years ago very little was known about maximal functions, today there are two basic approaches known which allow us to solve these problems. These are the «Fourier transform approach» and "covering lemma approach» which have been discussed in detail on many occasions (see Stein-Wainger [7], and Córdoba [8], R. Fefferman [9]). Rather than repeating already familiar themes, perhaps it would be more enlightening to mention the main unsolved problem in the area, and why the two techniques, as they stand, seem inadequate to settle this problem. Quite simply, we are unable to obtain results so far about maximal operators which are aunbounded on $L^{2}\left(R^{n}\right)$. (The main examples are Kakeya type maximal functions when $n \geqslant 3$ and the Stein spherical maximal function on $R^{2}$ ). It is clear that the obvious application of Fourier transform techniques will not work in such a context, because on the $L^{p}$ spaces we must deal with, the Plancherel theorem is not available. On the other hand, to prove an $L^{p}$ maxi- 
mal theorem via the covering lemma approach, we must consider estimating, for appropriate sets $\left\{R_{k}\right\}_{k=, 2, \ldots}$, the quantity

$$
\left\|\sum \chi_{R_{k}}\right\|_{L^{p^{\prime}}} \text { where } \frac{1}{p}+\frac{1}{p^{\prime}}=1 \text {. }
$$

We usually only know how to do this when $p^{\prime}$ is an integer $m$, since then we may expand $\left(\sum \chi_{R_{k}}\right)^{m}$, and finish by estimating quantities like $\mid R_{k_{1}} \cap R_{k_{2}} \cap \cdots \cap$ $\cap \boldsymbol{R}_{k_{m}} \mid$ (see Córdoba-R. Fefferman [10]). Unfortunately, when $2<p<\infty$, $1<p^{\prime}<2$, hence $p^{\prime}$ will be non-integral, and this technique cannot be applied. Whether a third, totally different approach will be required to solve such problems, or just a clever twist of the existing machinery, only time will tell.

Let us now retreat a little, and go back to the simplest case of a multiparameter problem, the case of product spaces. Before doing this we shall remind the reader of two theorems of enormous importance from the classical theory.

(1) $\left(H^{1}\left(R^{1}\right)\right)^{*}=\operatorname{BMO}\left(R^{1}\right)$. This theorem of C. Fefferman characterizes those functions $\phi(x)$ which act, by integration, continously on $H^{1}\left(R^{1}\right)=$ $=\left\{f \in L^{1}\left(R^{1}\right) \mid H(f) \in L^{1}\left(R^{1}\right)\right\}$. Such $\phi$ are characterized by the estimate

$$
\frac{1}{|I|} \int_{I}\left|\phi(x)-\phi_{I}\right|^{2} d x \leqslant C
$$

for all intervals $I$, and $C$ independent of $I$. (Here $\phi_{I}$ is the mean value of $\phi(x)$ over I) [11].

(2) The Atomic Decomposition of $H^{p}\left(R^{1}\right), p \leqslant 1$. Coifman [12] showed that every function $f \in H^{p}\left(R^{1}\right)$ could be written in the form $f=\sum_{k=1} \lambda_{k} a_{k}$ where $\lambda_{k}$ are scalars satisfying $\sum\left|\lambda_{k}\right|^{p} \leqslant C\|f\|_{H^{p\left(R^{1}\right)}}^{p}$ and the $a_{k}$ are functions known as $H^{p}$ atoms. An atom is a function $a(x)$ supported on an interval $I$ such that a certain number, $N(p)$, of its moments vanish,

$$
\int_{I} a(x) x^{k} d x=0 \quad \forall k \leqslant N(p) \text { and }\|a\|_{L^{2}} \leqslant|I|^{1 / 2-1 / p} .
$$

(We shall not be concerned here about the exact value of $N(p)$, except to remark that as $p \rightarrow 0, N(p) \rightarrow \infty$ and for $p$ close enough to 1 , we may take $N(p)=0)$.

Using these theorems, as we all know, enables us to obtain trivially results which, prior to their discovery, were either unproven or else most difficult to prove.

What we shall consider here is the theory, on $R^{2}$, of operators like the double Hilbert transform $H_{1} H_{2} f=f *\left(1 / x_{1} x_{2}\right)$, which are singular integrals with kernels having one dimensional singularity sets. It is trivial to observe that $H_{1} H_{2}$ is a bounded operator on $L^{p}\left(R^{2}\right)$ when $1<p<\infty$, by an iteration argument. We might ask whether there is an $H^{p}$ theory for $p \leqslant 1$ which cor- 
responds to the double Hilbert transform in the same way that classical $H^{p}$ theory on $R^{1}$ corresponds to the ordinary Hilbert transform. Thanks to the efforts of Gundy and Stein [13], there is such a theory, which we shall now describe. Let us, in analogy to classical Hardy space theory set $H^{1}\left(R^{1} \times R^{1}\right)=$ $=\left\{f \in L^{1}\left(R^{2}\right) \mid H_{1} f, H_{2} f\right.$, and $\left.H_{1} H_{2} f \in L^{1}\left(R^{2}\right)\right\}$. Then if $S(f)$ denotes the Lusin area integral of the biharmonic extension, $u$, of $f$ to $R_{+}^{2} \times R_{+}^{2}$, and $f^{*}$ denotes the non-tangential maximal function of $u$, then according to the Gundy-Stein theorem $f \in H^{1}\left(R^{1} \times R^{1}\right) \Leftrightarrow S(f) \in L^{1}\left(R^{2}\right) \Leftrightarrow f^{*} \in L^{1}\left(R^{2}\right)$. According to this theorem, $S(f) \in L^{p}\left(R^{2}\right)$ is equivalent to $f^{*} \in L^{p}\left(R^{2}\right)$ for all $p>0$, so that we may define $H^{p}\left(R^{1} \times R^{1}\right)$ as the set of all $f$ so that $f^{*}$ or $S(f) \in L^{p}\left(R^{2}\right), p>0$. At this point we come to a very important question. Do we have simple analogues, in this product space setting, of the two theorems above on $H^{1}$-BMO duality and on the atomic decomposition of $H^{p}$ spaces?

There is an obvious guess as to what the BMO space and atoms should look like. Take the case of an $H^{1}\left(R^{1} \times R^{1}\right)$ atom. The simplest example of such an object should be functions of the form $a_{1}\left(x_{1}\right) a_{2}\left(x_{2}\right)$ where each $a_{i}$ is an $H^{1}\left(R^{1}\right)$ classical atom. Of course such functions satisfy:

(1) $a\left(x_{1}, x_{2}\right)$ is supported in a rectangle $R=I \times J$ with $\|a\|_{L^{2}(R)} \leqslant|R|^{-1 / 2}$ and

(2) $\int_{I} a\left(x_{1}, x_{2}\right) d x_{1}=0 \quad \forall x_{2} \in J$ $\int_{J} a\left(x_{1}, x_{2}\right) d x_{2}=0 \quad \forall x_{1} \in I$.

Such a function (for reasons which we shall mention shortly) will be called an $H^{1}\left(R^{1} \times R^{1}\right)$ rectangle atom. To define $H^{p}$ rectangle atom, we simply change $|R|^{-1 / 2}$ in (1) above to $|R|^{1 / 2-1 / p}$, and require an appropriate number of moments of $a$ in each variable separately to vanish. (If $p$ is sufficiently close to 1 the vanishing of the mean value in (2) is sufficient).

Now a very important conjecture was that every $f \in H^{1}\left(R^{1} \times R^{1}\right)$ could be written as an absolutely convergent sum of such rectangle atoms. Along with this conjecture, was the companion which asked whether the dual space of $H^{1}\left(R^{1} \times R^{1}\right)$ (call it $\mathrm{BMO}\left(R^{1} \times R^{1}\right)$ ) could be characterized as those functions $\phi \in L_{\mathrm{loc}}^{2}\left(R^{2}\right)$ such that for each rectangle $R$, there exist functions $\phi_{1}^{R}\left(x_{1}\right)$ and $\phi_{2}^{R}\left(x_{2}\right)$ so that

$$
\frac{1}{|R|} \int_{R}\left|\phi\left(x_{1}, x_{2}\right)-\phi_{1}^{R}\left(x_{1}\right)-\phi_{2}^{R}\left(x_{2}\right)\right|^{2} d x_{1} d x_{2} \leqslant C .
$$

These conjectures where both disproven by Lennart Carleson [14] in 1974, and for some time it appeared that there could be no simple $H^{p}$ and BMO theory for product spaces.

Somewhat later, Alice Chang and R. Fefferman [15] gave an atomic decomposition of $H^{p}\left(R^{1} \times R^{1}\right)$, for $0<p \leqslant 1$, and a characterization of BMO in 
which the role of rectangie was, to some extent replaced by arbitrary open sets of finite measure. Let us be specific and give the precise definition of an $H^{1}\left(R^{1} \times R^{1}\right)$ atom $a$. Suppose $\Omega$ is an open set of finite measure in $R^{2}$ and $\mathcal{R}(\Omega)$ denotes the family of all maximal dyadic subrectangles of $\Omega$. An $H^{1}$ atom $a$ is a function supported in $\Omega$ satisfying $\|a\|_{L^{2}} \leqslant|\Omega|^{-1 / 2}$ and $a$ can be written as $a=\sum_{R \in \mathcal{R}(\Omega)} \alpha_{R}$ where $\alpha_{R}$ where $\alpha_{R}$ is supported in the double of $R$, satisfies $\int_{I} \alpha_{R}\left(x_{1}, x_{2}\right) d x_{1}=\int_{J} \alpha_{R}\left(x_{1}, x_{2}\right) d x_{2}=0$ where the double of $R$ is $I \times J$, and $\left(\Sigma_{R}\left\|\alpha_{R}\right\|_{L^{2}}^{2}\right)^{1 / 2} \leqslant|\Omega|^{-1 / 2}$. Chang-Fefferman [15] also gave a characterization of $\operatorname{BMO}\left(R^{1} \times R^{1}\right)$ corresponding to this atomic decomposition, and proved interpolation results [16] between these $H^{p}$ spaces for $p<1$ and the spaces $L^{p}$ for $p>1$. These results have a number of applications, and we just mention briefly that as one of them, we have a real variable proof that if for some $f, S(f) \in L^{p}, p \leqslant 1$, then $f^{*} \in L^{p}$, which Gundy and Stein proved using the theory of analytic functions of a complex variable or Brownian motion probabilistic arguments.

The main applications of this $H^{p}$ and BMO theory were discovered quite recently by J. L. Journé [17], whose work we shall describe below. Journé was interested in two types of product singular integral results. The first was the problem of obtaining $L^{2}$ estimates for bi-commutator integrals. Here, we shall state a special case of his result. Suppose that $\left(\partial^{2} A / \partial x_{1} \partial x_{2}\right)\left(x_{1}, x_{2}\right) \in L^{\infty}\left(R^{2}\right)$. Suppose we set $\tilde{A}\left(x_{1}, x_{2}, y_{1}, y_{2}\right)=A\left(y_{1}, y_{2}\right)-A\left(x_{1}, y_{2}\right)-A\left(y_{1}, x_{2}\right)+A\left(x_{1}, x_{2}\right)$ and

$$
T_{k} f\left(x_{1}, x_{2}\right)=\iint_{R^{2}} \frac{\left[\tilde{A}\left(x_{1}, x_{2}, y_{1}, y_{2}\right)\right]^{k} f\left(y_{1}, y_{2}\right)}{\left(x_{1}-y_{1}\right)^{k+1}\left(x_{2}-y_{2}\right)^{k+1}} d y_{1} d y_{2} .
$$

Then the bicommutator $T_{k}$ is bounded on $L^{2}\left(R^{2}\right)$ for $k \geqslant 1$. This is proved via a «T1-theorem» which is stated in terms of the space $\mathrm{BMO}\left(R^{1} \times R^{1}\right)$ as discussed above.

The second result Journé considers is the generalization to two dimensions of J. L. Rubio de Francia's theorem on Littlewood-Paley theory. Suppose that $\rho_{k}$ is a sequence of disjoint rectangles in $R^{2}$ and for each $k$ let $S_{k} \hat{f}(\xi)=\chi_{\rho_{k}}(\xi) \hat{f}(\xi)$. Then Journé shows that $\left\|\left(\sum\left|S_{k} f\right|^{2}\right)^{1 / 2}\right\|_{L^{p}} \leqslant C_{p}\|f\|_{L^{p}}$ for all $p \geqslant 2$.

The reader familiar with the one parameter analogues of the two theorems above realizes that they are both phrased in terms of $L^{2}$ or $L^{p}$ estimates, but they are really theorems about operators which map $L^{\infty}$ into BMO. It is not surprising then, that in order to show these results, Journé proves a general theorem about operators mapping $L^{\infty}$ boundedly to $\mathrm{BMO}\left(R^{1} \times R^{1}\right)$. To state his theorem precisely we require some notation. Suppose that $K(x, y)$ is a kernel with $x, y \in R^{1}$ and that the associated integral operator $S f(x)=\int_{R^{1}} K(x, y) f(y) d y$ is bounded on $L^{2}\left(R^{1}\right)$. Suppose that for some $\delta>0$ and for all $\gamma \geqslant 2$, 


$$
\int_{|y-x|>\gamma\left|x-x^{\prime}\right|}\left|K(x, y)-K\left(x^{\prime}, y\right)\right| d y \leqslant C \gamma^{-\delta} .
$$

Then define the norm \|\|$_{C Z}$ by $\|S\|_{C Z}=\|S\|_{L^{2}, L^{2}}+\inf \left\{\left.C\right|^{*}\right.$ holds $\}$. Now suppose $K\left(x_{1}, x_{2}, y_{1}, y_{2}\right)$ is defined for $x_{i}, y_{i} \in R^{1}$. Set $\tilde{K}^{1}\left(x_{1}, y_{1}\right)$ to be the operator on $R^{1}$ whose kernel is $\tilde{K}^{1}\left(x_{1}, y_{1}\right)\left(x_{2}, y_{2}\right)=K\left(x_{1}, x_{2}, y_{1}, y_{2}\right)$, and define $\tilde{K}^{2}$ similarly. The theorem in [17] is then: Let

$$
T f\left(x_{1}, x_{2}\right)=\iint_{R^{2}} K\left(x_{1}, x_{2}, y_{1}, y_{2}\right) f\left(y_{1}, y_{2}\right) d y_{1} d y_{2}
$$

and suppose $T$ is bounded on $L^{2}\left(R^{2}\right)$. Suppose further that

$$
\int_{\left|y_{1}-x_{1}\right|>\gamma\left|x_{1}-x_{1}^{\prime}\right|}\left\|\tilde{K}^{1}\left(x_{1}, y_{1}\right)-\tilde{K}^{1}\left(x_{1}^{\prime}, y_{1}\right)\right\|_{C Z} d y_{1} \leqslant C \gamma^{-\delta}
$$

and similarly for $\tilde{K}^{2}$. Then $T$ maps $L^{\infty}\left(R^{2}\right)$ boundedly to $\mathrm{BMO}\left(R^{1} \times R^{1}\right)$.

In proving this result, Journé introduces a beautiful covering lemma for rectangles, and we can state this in one of its forms as follows. Suppose $\Omega \subseteq R^{2}$ is an open set of finite measure.

Denote by $R_{2}(\Omega)$ those dyadic subrectangles of $\Omega$ which are maximal in the $x_{2}$ direction. Let $\tilde{\Omega}=\left\{M_{S}\left(\chi_{\Omega}\right)>1 / 2\right\}$ where $M_{S}$ is the strong maximal operator. For $R=I \times J \in \mathbb{R}_{2}(\Omega)$, suppose $S$ is the biggest dyadic interval containing $I$ so that $S \times J \subseteq \tilde{\Omega}$. Finally, set $\gamma_{1}(R)=|S / I|$. Then

$$
\sum_{R \in \mathcal{R}_{2}(\Omega)}|R| \gamma_{1}(R)^{-\delta} \leqslant C|\Omega| .
$$

A similar estimate holds reversing the roles of the $x_{1}$ and $x_{2}$ variables.

Also quite recently, R. Fefferman [18] has proven a theorem along these lines which states that in order to know the action of an operator on $L^{p}, H^{p}$, or $L \log L$, all we have to do is to check the action of that operator on a rectangle atom. In other words it is almost as if Carleson's counterexaple did not exist:

Theorem [18], [19]: Suppose that an operator $T$ is bounded on $L^{2}\left(R^{2}\right)$. Suppose further that if $a$ is a rectangle $H^{p}$ atom, $p \leqslant 1$, supported on a rectangle $R$ we have

$$
\int_{c_{\bar{R}_{\gamma}}}|T(a)|^{p} d x_{1} d x_{2} \leqslant C \gamma^{-\delta} \text { for all } \gamma \geqslant 2 .
$$

(Here $\tilde{R_{\gamma}}$ is the dilation of $R$ by a factor $\gamma$ concentric with $R$.) Then $T$ maps $H^{p}\left(R^{1} \times R^{1}\right)$ boundedly to $L^{p}\left(R^{1} \times R^{1}\right)$ and in the case $p=1$ we have

$$
\left|\left\{\left(x_{1}, x_{2}\right)|| x_{i}|<1,| T f\left(x_{1}, x_{2}\right) \mid>\alpha\right\}\right| \leqslant \frac{C}{\alpha}\|f\|_{L \log L} .
$$


The proof is totally trivial once we have both the $H^{p}$ atomic decomposition and the Journé covering lemma. (For $L \log L$ there is one extra twist. One uses an atomic decomposition for $L \log L$ see [19].) Let us give a rough outline of the proof to show that if $p=1, T$ maps $H^{1}$ boundedly to $L^{1}$. We take an atom $a$ with associated open set $\Omega$ and decomposition $a=\sum_{R \in \mathcal{R}(\Omega)} \alpha_{R}$. To each $R=I \times J$, associate the largest dyadic $\delta \supset I$ so that $\delta \times J \subseteq \tilde{\Omega}$. Let $R_{1}=\mathcal{S} \times J$. Then $R_{1} \in R_{1}(\tilde{\Omega})$, and let $\mathcal{J}$ be maximal containing $J$ so that $\mathcal{S} \times \mathcal{J} \subseteq \tilde{\tilde{\Omega}}$. Let $\hat{R}$ be the tenfold dilation of $\mathcal{S} \times \mathcal{J}$. Since $\hat{R} \subseteq \hat{\Omega}$ it is enough to show that

$$
\sum_{R} \int_{c_{\hat{R}}}\left|T\left(\alpha_{R}\right)\right| d x_{1} d x_{2} \leqslant C
$$

since

$$
\int_{\cup \mathcal{R}}|T(a)| d x_{1} d x_{2} \leqslant|\cup \hat{R}|^{1 / 2}\|T(a)\|_{L^{2}} \leqslant C|\Omega|^{1 / 2}|\Omega|^{-1 / 2}=C .
$$

We estimate each $\int_{c_{\hat{R}}}\left|T\left(\alpha_{R}\right)\right| d x_{1} d x_{2}$ in the most simple way. For instance

$$
\int_{x_{1} \notin \bar{S}}\left|T\left(\alpha_{R}\right)\right| d x_{1} d x_{2} \leqslant \gamma_{1}(R)^{-\delta}\left\|\alpha_{R}\right\|_{L^{2}}|R|^{1 / 2}
$$

by the hypothesis of the theorem (here $\widetilde{\delta}$ is the tenfold dilate of $\delta$ ). If we sum on $R$ and use Cauchy-Schwarz, we get

$$
\begin{aligned}
\sum_{R} \int_{x_{1} \notin \tilde{S}(R)}\left|T\left(\alpha_{R}\right)\right| d x_{1} d x_{2} & \leqslant \sum_{R}\left\|\alpha_{R}\right\|_{L^{2}}|R|^{1 / 2} \gamma_{1}(R)^{-\delta} \\
& \leqslant\left(\sum\left\|\alpha_{R}\right\|_{L^{2}}^{2}\right)^{1 / 2}\left(\sum|R| \gamma_{1}^{-2 \delta}(R)\right)^{1 / 2} \\
& \leqslant|\Omega|^{-1 / 2} \cdot C|\Omega|^{1 / 2}=C .
\end{aligned}
$$

The estimate of $\sum_{R} \int_{x_{2} \notin \tilde{\mathfrak{J}}}\left|T\left(\alpha_{R}\right)\right| d x_{1} d x_{2}$ is only a little bit trickier (see [18]), and finishes the proof.

Before finishing we should remark that there are a number of applications of the theorem above, and here we content ourselves with the statement of just one of them. This is joint work of R. Fefferman with K. C. Lin [20].

Let a bounded function $m(\xi, \eta)$ be given on $R^{n} \times R^{m}$ satisfying

$$
|\xi|^{|\alpha|}|\eta|^{|\beta|}\left|\partial_{\xi}^{\alpha} \partial_{\eta}^{\beta} m(\xi, \eta)\right| \leqslant C
$$

for all multi-indices $\alpha, \beta$ with $|\alpha| \leqslant[n / 2]+1,|\beta| \leqslant[m / 2]+1$. Let $T f(\xi, \eta)=$ $=m(\xi, \eta) \cdot \hat{f}(\xi, \eta)$. Then

$$
\left|\left\{(x, y) \in R^{n} \times R^{m}|| x|,| y|<1,| T f(x, y) \mid>\alpha\right\}\right| \leqslant C / \alpha\|f\|_{\left.L \log L^{+}(|x|,|y|) \leq 1\right)} .
$$

While there can be little doubt that much progress has been made in the area of multiple parameter Fourier analysis, it is also clear that most of the work 
is yet to come. Perhaps it can be said that this is the most central area of modern Fourier Analysis where our knowledge is at such a primitive level.

\section{References}

[1] Calderón, A.P., and Zygmund, A. On the Existence of Certain Singular Integrals, Acta Math., 88, 1952.

[2] Fefferman, C. The Multiplier Problem for the Ball, Annals of Math., 94, 1971.

[3] Carleson, L., and Sjölin, P. Oscillatory Integrals and a Multiplier Problem for the Disk, Studia Math. 44, 1972.

[4] Fefferman, C. A Note on Spherical Summation Multipliers, Israel Journal of Math̆., 1973.

[5] Córdoba, A. A Note on Bochner-Riesz Operators, Duke Journal of Math., 1979.

[6] Córdoba, A and Fefferman, R. On the Equivalence Between the Boundedness of Certain Classes of Maximal and Multiplier Operators in Fourier Analysis, PNAS, 74, 1977.

[7] Stein, E. M. and Wainger, S. Problems in Harmonic Analysis Relted to Curvature, Bulletin of the $A M S, 84,1978$.

[8] Córdoba, A. Maximal Functions, Covering Lemmas, and Fourier Multipliers, Proceedings of Symp. in Pure Math., Vol. 35, 1979.

[9] Fefferman, R. Covering Lemmas, Maximal Functions, and Multipliers in Fourier Analysis, Proceedings of Symp. in Pure Math., Vol. 35, 1979.

[10] Córdoba A. and Fefferman, R. On Differentition of Integrals, PNAS, 74, 1977.

[11] Fefferman, C. Characterization of Bounded Mean Oscillation, Bulletin AMS, 77, 1971.

[12] Coifman, R. R. A Real Variable Characterization of $H^{p}$, Studia Math., 51, 1974.

[13] Gundy, R. and Stein, E. M. $\quad H^{p}$ Theory for the Polydisk, PNAS, 76, 1979.

[14] Carleson, L. A Counterexample for Measures Bounded on $H^{p}$ for the Bidisc, Mittag-Leffler Report No. 7, 1974.

[15] Chang, A. and Fefferman, R. A Continuous Version of Duality of $H^{1}$ with BMO on the Bi-Disc, Annals of Math. 112, 1980.

[16] Chang, A. and Fefferman, R. The Calderón-Zygmund Decomposition on Product Domains, American Journal of Math., 104, 1982.

[17] Journé, J.L. Calderón-Zygmund Operators on Product Spaces, Rev. Mat. Iberoamericana, 3, 1985.

[18] Fefferman, R. Calderón-Zygmund Theory for Product Domains, to appear in PNAS.

[19] Fefferman, R. Note on a Lemma of Zo, to appear.

[20] Fefferman, R., and Lin, K. C. to appear.

R. Fefferman,

University of Chicago

Chicago, IL 60637

U.S.A. 